\title{
The Charge-Mass-Spin Relation of Clifford Polyparticles, Kerr-Newman Black Holes and the Fine Structure Constant
}

\author{
Carlos Castro \\ Center for Theoretical Studies of Physical Systems Clark Atlanta University, Atlanta, GA. 30314
}

A Clifford-algebraic interpretation is proposed of the charge, mass, spin relationship found recently by Cooperstock and Faraoini which was based on the Kerr-Newman metric solutions of the Einstein-Maxwell equations. The components of the polymomentum associated with a Clifford polyparticle in four dimensions provide for such a charge, mass, spin relationship without the problems encountered in Kaluza-Klein compactifications which furnish an unphysically large value for the electron charge. A physical reasoning behind such charge, mass, spin relationship is provided, followed by a discussion on the geometrical derivation of the fine structure constant by Wyler, Smith, Gonzalez-Martin and Smilga. To finalize, the renormalization of electric charge is discussed and some remarks are made pertaining the modifications of the charge-scale relationship, when the spin of the polyparticle changes with scale, that may cast some light into the alleged Astrophysical variations of the fine structure constant.

Key words : Clifford Algebras; Black Holes; Dirac electron.

PACS : 11 E88, 15 A66, 83 C57

\section{INTRODUCTION}

\subsection{CLIFFORD ALGEBRAS IN PHYSICS}

Clifford algebras display a rich mathematical structure which is very useful for a unified description of geometry and physics $[1,2,3,4,5,6,9]$. For example, a geometric approach to the physics of the Standard Model in terms of Clifford algebras was advanced by [3] . Important applications have also been found in string theory, extended objects, gravity, QFT.... Using Clifford-algebraic methods, and related ideas, the bosonic $p$-brane propagator, in the quenched-reduced minisuperspace approximation, was constructed in [10]; the logarithmic corrections to the black hole entropy based on the geometry of Clifford space were obtained in [12]; a higher derivative gravity action with torsion from a geometric action was derived starting from the scalar curvature in Clifford-spaces [11]. Many recent important aspects of Clifford algebra are described in the monographs [5]. 
In $[7,8,9]$ it was proposed that every physical quantity could in fact be assigned to a polyvector, or multivector, that is a Clifford number or a Clifford aggregate. Since spinors are the members of left or right minimal ideals of Clifford algebra they may provide the framework for a deeper understanding of sypersymmetries, i.e., the transformations relating bosons and fermions. The Fock-Stueckelberg theory of relativistic particle can be embedded in the Clifford algebra of spacetime [ 9 ] . In this new physical theory the arena for physics is no longer the ordinary spacetime, but a more general Clifford manifold of Clifford algebra valued objects, polyvectors. Such a manifold has been called a pan-dimensional continuum [8] or $C$-space [7]. The latter describes on a unified basis the objects of various dimensionality: not only points, but also closed lines, surfaces, volumes,..., called 0-loops (points), 1-loops (closed strings) 2-loops (closed membranes), 3-loops, etc.. It is a sort of a dimension category, where the role of functorial maps is played by C-space transformations which reshuffles a $p$-brane history for a $p^{\prime}$-brane history or a mixture of all of them, for example.

Technically those transformations in C-space that reshuffle objects of different dimensions are generalizations of the ordinary Lorentz transformations, that mix space with time, to $C$-space. Since Clifford algebras contain the appropriate algebro-geometric features to implement this principle of polydimensional transformations and are deeply ingrained in the algebraic properties of quaternions and octonions [17], it is very plausible that this may lead to the proper formulation of string and $M$ theory [11] within the framework of Projective Geometry [24] . Clifford algebras implement naturally the idea of electric magnetic duality and incorporate Dirac monopoles in a straightforwad fashion [26]. Other recent approaches to the generalization of Maxwell Electrodynamics has been based in the study of gerbes or higher-gauge theories [27]. For a detail discussion of the role of Clifford algebras in Electrodynamics see [4,28].

The outline of this work goes as follows. In section $\mathbf{1}$ we briefly review the basic concepts of C-space Relativity and the use of polyvectors, or Clifford-valued objects, and describe how to generalize Maxwell's theory of Electrodynamics asociated with ordinary point-charges to a Generalized Maxwell theory in Clifford spaces involving extended charges and p-forms of arbitrary rank [25]. In section 2 we show that an interpretation of the charge, mass, spin relationship found recently by [13], based on a Kerr-Newman metric solution, can be given in terms of the components of the polymomentum of a Clifford polyparticle in four-dimensions without the problems encountered in Kaluza-Klein compactifications which furnish an unphysically large value for the electron charge. A short discussion of the the Dirac electron as a Kerr-Newman solution follows [14]. In section 3 we add some important comments related to the geometrical derivation of the observed numerical values of the fine structure constant within the context of Clifford algebras. In the final section, the Renormalization of electric charge with distance is discussed and some final remarks are made pertaining 
the modifications of the charge-scale relationship when the spin of the polyparticle changes with scale. This will cast some light in the alleged astrophysical variations of the fine structure constant [ 30 ] .

\subsection{THE EXTENDED RELATIVITY IN C-SPACE}

In order to generalize Maxwell theory of Electrodynamics we review briefly the extended relativity theory in C-spaces $[7,9,11]$ by starting with a natural generalization of the notion of a space-time interval in Minkowski space $d s^{2}$ to $\mathrm{C}$-space. This is given by the scalar part of the Clifford geometric product of two polyvectors :

$$
\|d X\|^{2}=<d X^{\dagger} d X>_{\text {scalar }}=d \Omega^{2}+d x_{\mu} d x^{\mu}+d x_{\mu \nu} d x^{\mu \nu}+\ldots
$$

where the operation $X^{\dagger}$ is the analog of the matrix transpose ( hermitian ) conjugation operation and represents the reversed Clifford polyvector obtained by reversing the ordering position of all the basis vectors $\gamma^{\mu}$ present in the expansion of $X$.

The Clifford valued polyvector:

$$
X=X^{M} E_{M}=\Omega 1+x^{\mu} \gamma_{\mu}+x^{\mu \nu} \gamma_{\mu} \wedge \gamma_{\nu}+\ldots
$$

denotes the position of a polyparticle in a manifold, called Clifford space or $C$-space.

The coordinates $x^{\mu_{1} \mu_{2}}, x^{\mu_{1} \mu_{2} \mu_{3}}, \ldots$ are the holographic areas, volumes, ...projections of the nested family of $p$-loops ( closed p-branes ) onto the embedding $D$-dim spacetime coordinate planes/hyperplanes and where the values of $p$ range from $: p=0,1,2,3, \ldots . . p_{\max }$. The maximal value $p_{\max }$ is $D-2$ since the $p_{\max }+1$ dimensional world-tube (the higher-dim version of a world-line) swept by the evolution of the closed $p_{\text {max }}{ }^{-}$ brane is being embedded into a $p_{\max }+2=D$ dimensional region. The interior of the world-tube is $D$-dim and its boundary is $D-1=p_{\max }+1$ dimensional.

For example, in $D=10$, one has that the maximal value $p_{\max }$ associated with the nested hierarchy of closed p-branes ( p-loops ) is given by $p(\max )=D-2=10-2=8$. Therefore, we have a nested-hierarchy of closed p-branes ( p-loops ) living in $D=10$ whose values of $p$ range from $p=0,1,2,3, \ldots \ldots . .7,8$ corresponding to points, closed strings, closed membranes ...closed 8-branes living in $D=10$ spacetime dimensions. In our case when $D=4$ we will have then point-particles, closed-strings and closed-membranes.

Therefore, the quantity $\Omega$ (a Lorentz scalar) is associated with the proper $p_{\max }+1$-dim region associated with the lateral hypersurface of the $p_{\max }+1$-dim world-tube( "cylinder") swept by the motion of a closed $p_{\text {max }}$-brane in the embedding target spacetime background. In order to match units in the expansion of the polyvector $X$ it is required to introduce a length scale. Such scale was chosen to be the minimal Planck scale $[7,16]$ and can be set to unity. 
For example, if we take differential $d X$ of $X$ and compute the scalar part of $\left\langle d X^{\dagger} d X>\right.$ we obtain the C-space analog of the particle proper time interval :

$$
d \Sigma^{2}=(d \Omega)^{2}+\Lambda_{P}^{2 D-2} d x_{\mu} d x^{\mu}+\Lambda_{P}^{2 D-4} d x_{\mu \nu} d x^{\mu \nu}+.
$$

where in (3) one has introduced explicitly the Planck scale $\Lambda_{P}$ in order to fuse together objects of different dimensionality : 0-loops ( points ), 1-loops ( closed strings ),.., p-loops ( closed p-branes ). Einstein introduced the speed of light as a universal absolute invariant in order to "unite" space with time (to match units) in the Minkwoski space interval:

$$
d s^{2}=c^{2} d t^{2}-d x_{i} d x^{i}
$$

A similar unification is needed here to "unite" objects of different dimensions, such as $x^{\mu}, x^{\mu_{1} \mu_{2}}$, etc... The minimal Planck scale then emerges as another universal invariant in constructing an extended scale relativity theory in C-spaces $[7,16]$. To show that the Planck scale is a minimal distance requires the introduction of Quantum Mechanics [31].

To continue along the same path of special Relativity in Minkowski space, one considers the analog of Lorentz transformations in C-spaces which transform a polyvector $X$ into another polyvector $X^{\prime}$ given by $X^{\prime}=R X R^{-1}$ with

$$
R=e^{\theta^{A} E_{A}}=\exp \left[\left(\theta I+\theta^{\mu} \gamma_{\mu}+\theta^{\mu_{1} \mu_{2}} \gamma_{\mu_{1}} \wedge \gamma_{\mu_{2}} \ldots .\right)\right]
$$

and

$$
R^{-1}=e^{-\theta^{A} E_{A}}=\exp \left[-\left(\theta I+\theta^{\nu} \gamma_{\nu}+\theta^{\nu_{1} \nu_{2}} \gamma_{\nu_{1}} \wedge \gamma_{\nu_{2}} \ldots . .\right)\right] . \quad R R^{-1}=1
$$

where the theta parameters:

$$
\theta ; \theta^{\mu} ; \theta^{\mu \nu} ; \theta^{\mu \nu \rho} \ldots
$$

are the C-space version of the Lorentz rotations/boosts parameters. The ordinary Lorentz algebra generator $L_{\mu \nu}$ can be written in terms of the commutator $\left[\gamma_{\mu}, \gamma_{\nu}\right]$.

Under these transformations the norm-squared of a polyvector $X$ given by the scalar part of the product $\left\langle X^{\dagger} X\right\rangle_{\text {scalar }}$ remains invariant. Since a Clifford algebra admits a matrix representation, one can represent the norm of polyvectors $\left\langle X^{\dagger} X\right\rangle_{\text {scalar }}$ in terms of the trace operation as: $\|X\|^{2}=$ Trace $X^{2}$ Hence under C-space Lorentz transformation the norms of polyvectors behave like follows:

$$
\text { Trace }\left(X^{\prime}\right)^{2}=\text { Trace }\left[R X^{2} R^{-1}\right]=\text { Trace }\left[R R^{-1} X^{2}\right]=\text { Trace } X^{2} \text {. }
$$


These norms are invariant under C-space Lorentz transformations due to the cyclic property of the trace operation and $R R^{-1}=1$. This completes the very brief review of relativity in C-spaces [ 7, 9, 11$]$.

A C-space extension of Maxwell's Electrodynamics has been studied in [25] by starting with the differential operator in C-spaces which is the generalized Dirac operator:

$$
d=E^{M} \partial_{M}=1 \partial_{\Omega}+\gamma^{\mu} \partial_{x_{\mu}}+\gamma^{\mu} \wedge \gamma^{\nu} \partial_{x_{\mu \nu}}+\ldots
$$

the indices $M, N \ldots$ run over all the polyvector basis elements and range from $1,2 \ldots . .2^{D}$ since a Clifford algebra in $D$-dim has $2^{D}$ basis elements. At this point we will not be concerned with the representations of Clifford algebras in $(r, s)$ dimensions. Where $r, s$ denotes the temporal and spatial dimensions respectively [ 2 ] .

The generalized Maxwell field strength in C-space is :

$$
\begin{gathered}
F=d A=E^{M} \partial_{M}\left(E^{N} A_{N}\right)=E^{M} E^{N} \partial_{M} A_{N}=\frac{1}{2}\left\{E^{M}, E^{N}\right\} \partial_{M} A_{N}+ \\
\frac{1}{2}\left[E^{M}, E^{N}\right] \partial_{M} A_{N}=\frac{1}{4} F_{(M N)}\left\{E^{M}, E^{N}\right\}+\frac{1}{4} F_{[M N]}\left[E^{M}, E^{N}\right] .
\end{gathered}
$$

where one has decomposed the Field strength components into a symmetric plus antisymmetric piece by simply writing the Clifford geometric product of two polyvectors $E^{M} E^{N}$ as the sum of an anticommutator plus a commutator piece respectively .

$$
\begin{aligned}
& F_{(M N)}=\frac{1}{2}\left(\partial_{M} A_{N}+\partial_{N} A_{M}\right) . \\
& F_{[M N]}=\frac{1}{2}\left(\partial_{M} A_{N}-\partial_{N} A_{M}\right) .
\end{aligned}
$$

A measure of integration in C-space can be written as:

$$
[\mathcal{D} X]=(d \Omega)\left(d x^{0} d x^{1} \ldots d x^{D}\right)\left(d x^{01} d x^{02} . .\right)\left(d x^{012} \ldots\right) \ldots \ldots\left(d x^{0123 \ldots D}\right)
$$

The standard C-space Maxwell action is up to a numerical factor given by :

$$
S(A)=\int[\mathcal{D} X] F_{[M N]} F^{[M N]} .
$$

and is automatically gauge invariant under the transformations $\delta A_{M}=\partial_{M} \Lambda$ since $\delta F_{[M N]}=0$ due to the antisymmetry condition $\left[\partial_{M}, \partial_{N}\right] \Lambda=0$.

The action $[25,31]$ 


$$
\int[\mathcal{D} X]<F^{\dagger} F>_{\text {scalar }}=\int[\mathcal{D} X]\left(F_{(M N)} F^{(M N)}+F_{[M N]} F^{[M N]}\right) .
$$

is invariant ( up to total derivatives) under infinitesimal gauge transformations provided the symmetric part of $F$ is divergence-free $\partial_{M} F^{(M N)}=0[25]$.

It was later noticed in [31] that this divergence-free condition has the same effects as if one were fixing a gauge leaving a residual symmetry of restricted gauge transformations such that the gauge symmetry parameter obeys the Laplace-like equation $\partial_{M} \partial^{M} \Lambda=0$. Such residual ( restricted ) symmetries are precisely those that leave invariant the divergence-free condition on the symmetric part of $F$. Residual, restricted symmetries occur, for example, in the light-cone gauge of p-brane actions leaving a residual symmetry of volume-preserving diffs and in string theory, in the conformal gauge, leaving a residual symmetry under conformal reparametrizations; i.e. the Virasoro algebras whose symmetry transformations are given by holomorphic and anti-holomorphic reparametrizations of the string world-sheet.

This Laplace-like condition on the gauge parameter is also the one required such that the action (14b) is invariant under finite (restricted) gauge transformations [31] since under such (restricted) finite transformations the Lagrangian (14b) changes by second-order terms of the form $\left(\partial_{M} \partial_{N} \Lambda\right)^{2}$, which are total derivatives if, and only if, the gauge parameter is restricted to obey the analog of Laplace equation $\partial_{M} \partial^{M} \Lambda=0$ [31]. Concluding, the effects of adding the symmetric pieces of $F$ to the action leads to a restricted gauge symmetry which has the same effects as if one were fixing a gauge $\partial_{M} F^{(M N)}=0$ [31].

The matter-field minimal coupling is :

$$
\int A_{M} d X^{M}=\int[\mathcal{D} X] A_{M} J^{M}
$$

where one has reabsorbed the coupling constant, the C-space analog of the electric charge, within the expression for the $A$ field itself and the current $J$ polyvector is proportional to the polymomentum, like in ordinary EM the current four-vector $J^{\mu}$ is proportional to the four-velocity with a delta function proportionality factor:

$$
\delta\left(x^{0}-x^{0}(\tau)\right) \delta\left(x^{1}-x^{1}(\tau)\right) \delta\left(x^{2}-x^{2}(\tau)\right) \delta\left(x^{3}-x^{3}(\tau)\right)
$$

which implies that the current has support along the worldline of the particle $x^{\mu}=x^{\mu}(\tau)$.

This matter-field coupling term (15) has the same form as the coupling of p-branes (whose world volume is $p+1$-dimensional) to antisymmetric tensor fields of rank $p+1$. The gauge-invariant equations of motion for the antisymmetric part of $F$ are the usual ones $\partial_{M} F^{[M N]}=J^{N}$. For further details about equations of 
motion, the construction of Noether currents, continuity equation, etc... see [25, 32]. This completes the brief review of C-space Relativity and generalized Maxwell's Electrodynamics in C-spaces.

\subsection{THE COMPONENTS OF THE POLYMOMENTUM}

With this preamble on Clifford spaces and polyvectors, we may continue with the physical interpretation of the polymomentum components of a polyparticle which will allow us to provide a Clifford-algebraic interpretation of the charge, mass and spin relationship based on the Kerr-Newman solutions to the EinsteinMaxwell equations [13].

Based on our basic postulate that a hierarchy of extended objects, a nested collection of p-loops, is a physical example of a Clifford polyparticle [ 7,11,32], we shall derive the relationship among charge, mass and spin from the geometry of C-spaces by using Clifford algebras. We will follow the nomenclature of chaper two in Pavsic's book [ 9 ] to describe the polymomentum components of a polyparticle in a $D=4$-dimensional spacetime. In the special case when one imposes the standard mass-shell condition of ordinary point-particle relativistic dynamics :

$$
p^{\mu} p_{\mu}-m^{2}=0
$$

it follows then that the invariant norm-squared of the polymomentum $P$, a Clifford-valued object $P=$ $P^{M} E_{M}$, associated with the polyvector-valued C-space coordinate $X=X^{M} E_{M}$ studied earlier, becomes in this 4 -dim case [9]:

$$
\begin{gathered}
P^{2}=P^{M} P_{M}=\kappa^{2}+\pi_{\mu} \pi^{\mu}+p_{\mu} p^{\mu}-m^{2}-\frac{1}{2} m_{P}^{2} S^{\mu \nu} S_{\mu \nu}=\mathcal{M}^{2} \Rightarrow \\
P^{2}=P^{M} P_{M}=\kappa^{2}+\pi_{\mu} \pi^{\mu}-\frac{1}{2} m_{P}^{2} S^{\mu \nu} S_{\mu \nu}=\mathcal{M}^{2} .
\end{gathered}
$$

where we have used the decomposition of a polymomentum as indicated by [ 9 ] :

$$
P=\kappa 1+p^{\mu} \gamma_{\mu}+S^{\mu \nu} \gamma_{\mu} \wedge \gamma_{\nu}+\pi^{\mu} \gamma_{5} \gamma_{\mu}+m \gamma_{5}
$$

The components of the polymomentum are all dynamical variables which are functions of the C-space analog of the proper time, $\Sigma$, like the four-momentum of an ordinary massive point particle given by $p^{\mu}=$ $m d x^{\mu} / d \tau$ is a function of the proper time parameter $\tau$. This means that in C-space, $m$ is a truly dynamical variable and the constraint $P^{2}=\mathcal{M}^{2}$ is the C-space version of the ordinary mass-shell constraint $p_{\mu} p^{\mu}=m^{2}$ resulting from reparametrization invariance. 
It has been shown in [9] that the constrained dynamics in C-space, encoded in the constraint $P^{2}=\mathcal{M}^{2}$, leads to an unconstrained particle dynamics in ordinary spacetime such that a C-space polyparticle action leads naturally to the unconstrained Stueckelberg action in spacetime. Therefore, the quantity $\mathcal{M}$ must not be confused with the dynamical variable $m$ which is another one of the components of the polymomentum.

In $D=4$, the maximal value of $p$ associated with the nested hierarchy of p-loops ( closed p-branes ) is $p_{\max }=D-2=4-2=2$. It corresponds to a closed-membrane such that $\kappa$ is the conjugate-momentum variable to the proper $2+1$-dimensional region $\Omega$ associated with the (lateral) hypersurface of the worldtube swept by the evolution of a closed-membrane in a target 4-dim spacetime background [7]. The latter $2+1$-dim proper hypersurface $\Omega$ is a Lorentz scalar which is proportional to the Dirac-Nambu-Goto 2-brane (closed-membrane) action. The constant of proportionality is provided by the 2-brane tension.

As we said earlier, a closed string spans a two-dim tube (cylinder). A closed membrane spans a threedim world-tube, etc.... Spatial $p$-loops have a topology of the p-sphere $S^{p}$, and for $p=p_{\max }$ they enclose an interior $p_{\max }+1$-dim region. Including the time-coordinate, a clock, this brings the overall dimension of the embedding target spacetime to $D=p_{\max }+2=2+2=4$; i.e. the $2+1$-dim world-tubes are embedded in a spacetime of $D=2+2$ dimensions. The interior region of the world-tube is 4 dimensional. We shall not be concerned in this work with p-loop-instantons embedded in p+1-dimensions and other exotic p-loops [31].

Thus, the units of $\kappa$ associated with a closed-membrane $\left(p_{\max }=2\right)$ spanning a 3 -dim world-tube embedded in a 4-dim target spacetime background are those of (mass $)^{3}$. However, in the following, for convenience purposes, we shall fix the units of $\kappa$ to be those of an ordinary momentum, by an appropriate rescaling of all the variables by suitable powers of the Planck mass, $m_{P}$. Hence the units of $\kappa$ will be chosen to be those of mass, however we should keep in mind that the units of $\kappa$ are related to an energy per unit area; i.e. the same units as those of a membrane-tension : $(\text { mass })^{3}$.

The quantity $\pi_{\mu}$, after rescaled by powers of $\left(m_{P}\right)^{2}$, is proportional to the axial-vector momentum component of the polymomentum [9] and which can also be written in $D=4$ as the dual of the 3-vector :

$$
\left(m_{P}\right)^{2} \pi_{\mu}=\frac{1}{3 !} \epsilon_{\mu \nu \rho \sigma} p^{\nu \rho \sigma}
$$

The physical interpretation of the quantities $p^{\nu \rho \sigma}$ in (20a), which have units of $(\text { mass })^{3}$, is that they correspond to the "volume-momentum" conjugate variables of the three-dim ( spatial and timelike ) regions enclosed by the holographic projections of a 2-loop onto the embedding target spacetime hyperplanes. A 2-loop encloses a 3-dim interior region such that its ( spacelike and timelike ) holographic projections onto the 
hyperplanes of the embedding spacetime background are the $x^{\nu \rho \sigma}$ components of the polyvector $X$. Thus, the conjugate momentum variables to the latter components of $X$ are the volume-momentum $p^{\nu \rho \sigma}$ variables, with units of $(\text { mass })^{3}$, while its dual momentum is proportional the axial-vector momentum components $\left(m_{P}\right)^{2} \pi_{\mu}$. This clarifies the physical meaning of the axial-vector $\pi^{\mu}$ component of the polymomentum that we chose to have units of [mass] after rescaling all the components by suitable powers of $m_{P}$.

$p^{\mu}$ is timelike vector but the axial-vector $\pi^{\mu}$ is spacelike as shown by Pavsic [9] .

$$
\pi_{\mu} \pi^{\mu}<0 . \quad p_{\mu} p^{\mu}>0
$$

Since $\pi_{\mu}$ is spacelike we may set:

$$
\pi_{\mu} \pi^{\mu}=-\pi^{2}<0
$$

where one should not confuse the norm $\pi$ with the number $\pi$. Below we will see that the spacelike normsquared of the axial-vector $\pi^{\mu}$ given by $-\pi^{2}$ is the quantity that is related to the electric charge in the Kerr-Newman solutions.

$S^{\mu \nu} S_{\mu \nu}$ are the holographic area-momentum variables contribution to the norm-squared $P^{2}$. It has been shown by Pezzaglia [8] in his derivation of the Papapetrou's equations, and discussed further by Pavsic [9], that the conjugate-momentum to the holographic area-variables $x^{\mu \nu}$ are given by $S^{\mu \nu}$ and describe precisely the spin degrees of freedom of a polyparticle. In order to have the proper matching of mass-units for all the components, we have introduced a factor of the Planck mass $m_{P}$ in the $S^{\mu \nu} S_{\mu \nu}$ terms, likewise we introduced powers of the minimal Planck scale in the definition of the analog of the proper-time interval of a polyparticle in C-space. This concludes the physical interpretation of the components of the polymomentum associated with a polyparticle.

In the particular case when the mass parameter is set to be $\mathcal{M}=m_{P}$ and when the standard on-shell condition is imposed $p_{\mu} p^{\mu}-m^{2}=0$, eq-(18) becomes:

$$
P^{2}=\kappa^{2}+\pi_{\mu} \pi^{\mu}-m_{P}^{2} \frac{1}{2} S^{\mu \nu} S_{\mu \nu}=m_{P}^{2}
$$

where the Planck mass scale is $m_{P}=\sqrt{\hbar c / G}$. The definition of the spacelike condition of the axial-vector $\pi_{\mu} \pi^{\mu} \equiv-\pi^{2}$ in eq-(22) leads to:

$$
\kappa^{2}-\pi^{2}=m_{P}^{2}\left(1+S^{2}\right)
$$

after having defined $S^{2}=\frac{1}{2} S^{\mu \nu} S_{\mu \nu}$. 
The recently found relationship by [13] among charge, mass and spin, based upon a generalized definition of a Planck mass scale associated with the Kerr-Newman metric solutions of the Einstein-Maxwell equations, after equating the Compton scale with the horizon radius, is given by :

$$
m_{K N}=\frac{m_{P}}{\sqrt{2}} \sqrt{\frac{2\left(1+S^{2}\right)}{2-n^{2} e^{2}}} .
$$

where the quantized charge $q=n e$ is written in units of the electric charge . The fine structure constant in natural units $\hbar=c=1$ is given by $\alpha=e^{2}$.

For the sake of completeness, the detailed steps of [13] that leads to the charge, mass, spin relationship of the Kerr-Newman metric solution given by eq-(24) are reviewed in the appendix. It follows from eq-( 24$)$ :

$$
2 m_{K N}^{2}\left(1-\frac{n^{2} e^{2}}{2}\right)=m_{P}^{2}\left(1+S^{2}\right)
$$

Now we can finally match the functional form of eq-( 25$)$ with that of eq-( 23$)$ and conclude that:

$$
\begin{gathered}
\kappa^{2}-\pi^{2}=m_{P}^{2}\left(1+S^{2}\right)=2 m_{K N}^{2}\left(1-\frac{n^{2} e^{2}}{2}\right) . \Rightarrow \\
\kappa^{2}-\pi^{2}=\kappa^{2}\left(1-\frac{\pi^{2}}{\kappa^{2}}\right)=2 m_{K N}^{2}\left(1-\frac{n^{2} e^{2}}{2}\right) .
\end{gathered}
$$

We have the feedom in eq- $(26)$ to relate the extended Planck mass $m_{K N}$ to the $\kappa$ component of the polymomentum by setting $\kappa^{2}=2 m_{K N}^{2}$. Upon doing so one can infer from eq- $(26)$ that the spacelike axial-momentum component of the polymomentum $\pi^{\mu}$ obeys the relation:

$$
\frac{\pi^{2}}{\kappa^{2}}=\frac{\pi^{2}}{2 m_{K N}^{2}}=\frac{n^{2} e^{2}}{2} \Rightarrow \pi^{2}=-\pi_{\mu} \pi^{\mu}=\frac{\kappa^{2}}{2}\left(n^{2} e^{2}\right)=m_{K N}^{2} n^{2} e^{2}
$$

eqs- $(26,27)$ are the sought-after relations among charge, mass and spin.

\subsection{PHYSICAL INTERPRETATION OF THE CHARGE-MASS-SPIN RELATION}

We turn now to a physical interpretation of the relations behind eqs- $(26,27)$ and the differences between the conventional Kaluza-Klein compactification of $D=5$ (on a circle) down to $D=4$ dimensions . In the latter Kaluza-Klein compactifications the momentum component in the internal fifth dimension defines a new conserved quantity, due to the periodic motion in the internal circle of radius $R$, and which is quantized in discrete units like " charges ". In this case the quantized charges $q_{n}$ in integer units $n=1,2,3 \ldots$ of a fundamental charge $q_{K K}$ are related to the Kaluza-Klein momentum components $p_{5}=n / R$, in terms of $R$, the compactification radius of the internal circle. The charge internal-momentum relation goes as follows : 


$$
G\left(p_{5}\right)^{2}=\frac{\left(p_{5}\right)^{2}}{m_{\text {Planck }}^{2}}=\frac{n^{2}}{m_{\text {Planck }}^{2} R^{2}}=q_{n}^{2}=n^{2} q_{K K}^{2} .
$$

where $G$ is the gravitational constant in four-dim given by $G=L_{\text {Planck }}^{2}=\left(m_{\text {Planck }}\right)^{-2}$ in natural units of $\hbar=c=1$. The quantity $q_{K K}$ is the fundamental unit of charge in the Kaluza-Klein compactification.

For small radius compactifications $R$ of the order of the Planck scale, the value of $q_{K K}$ (in the corresponding units) is much greater than the electron charge $e$. Its value turns out to be extremely large since the magnitude of $p^{5}$ is of the order of the Planck mass. For a discussion of this Kaluza-Klein charge problem and how the use of Clifford polyvectors in $D=5$ dimensions may provide a plausible resolution, which is different from ours, we refer to Pavsic [9].

We don't have any longer the old problem which arises in Kaluza-Klein compactifications because the relevant point of our construction that relates charge, mass and spin given by eqs- $(26,27)$ is that we have not performed a Kaluza-Klein compactification in order to account for the origins of the electric charge since we are studying Clifford polyvectors based on Clifford algebras in $D=4$, and not in $D=5$.

Our relation obtained from eqs- $(26,27)$ yields also the charges $q^{2}=n^{2} e^{2}$ in terms of the ratios of the norms of two of the components of the polymomentum:

$$
\frac{2 \pi^{2}}{\kappa^{2}}=\frac{-2 \pi_{\mu} \pi^{\mu}}{\kappa^{2}}=n^{2} e^{2}
$$

but now the charges are different:

$$
\begin{gathered}
\frac{\left(p_{5}\right)^{2}}{m_{\text {Planck }}^{2}} \neq \frac{-2 \pi_{\mu} \pi^{\mu}}{\kappa^{2}} \Rightarrow \\
n^{2} q_{K K}^{2} \neq n^{2} e^{2} \Rightarrow q_{K K}^{2} \neq e^{2} .
\end{gathered}
$$

What we have found is that the norm-squared of the internal $p_{5}$ component of the momentum, in units of the Planck momentum $m_{P} c$, which yields the values of the charges in Kaluza-Kein compactifications, is not the same as the spacelike norm-squared of $\pi_{\mu}$ in units of $\kappa$. The advantage, now, is that the ratio of the norms of the components $\pi_{\mu} ; \kappa$ of the polymomentum associated with the Clifford algebra in four dimensions capture the same charge effects of the Kaluza-Klein compactifications without having an unphysically large value for the electron charge.

This idea of capturing the effects of higher dimensions has been applied recently by studying how Clifford algebras in four-dimensions encode the conformal algebra $S O(4,2)$ ( as a subalgebra) without the need to recur to six dimensions whatsoever [19]. From the Scale Relativistic point of view [22] the electric charge 
has been shown to be the eigenvalue of the scale-dilations operator associated with the spacetime resolution variables and which must not be confused with the ordinary dilations associated with the conformal group.

The physical meaning of the axial-momentum $\pi_{\mu}$ (times $\left.\left(m_{P}\right)^{2}\right)$ as the dual of the "volume-momentum" $p^{\nu \rho \sigma}$ conjugate variable to $x^{\nu \rho \sigma}$, was explained at the beginning of this section. The "volume-momentum" has units of a membrane tension $(\text { mass })^{3}$, or energy per unit area. It must be emphasized that upon restoring back the original units to all the components of the polymomentum, one can see that both $\left(m_{P}\right)^{2} \kappa$ and $p^{\nu \rho \sigma}$ have the same units since both of them are conjugate variables to 3-dim regions; i.e. the orginal units are those of energy per unit area, (mass) ${ }^{3}$, the same units of a membrane tension.

In the same fashion that one obtains the classical electron radius $r_{e}$ by equating the electrostatic energy $e^{2} / r_{e}$ to the electron's rest mass $m_{e}$ giving the relation $m_{e} r_{e}=e^{2}$, we have expressed in (29) the quantized charges $n^{2} e^{2}$ in terms of the ratios of the quantities $\pi^{2}$ and $\kappa^{2}$, which is just another statement of the condition that the energy per unit area of the membrane is a measure of the charge content enclosed by the three-dim region inside the membrane.

The (spinning) membrane-component of the polyparticle can be seen as a Kerr-Newman black-hole horizon whose tension (energy per unit area) encodes the charge content associated with the electromagnetic energy stored in the interior region. The connection between membranes and black-hole horizons is well known among the experts and has been coined " the membrane paradigm". Long ago, Dirac envisioned a classical electron as a membrane. In the next section we will discuss a very different view of the Dirac electron as a true naked singularity of the Kerr-Newman solution which is devoid of horizons, by definition. This concluds our discussion of the physical interpretation of the charge-mass-spin relations based on the four-dim Clifford algebra of a polyparticle.

Having described the mass, charge and spin in terms of a four-dim Clifford algebra, one could study higher-dim Clifford algebras with the hope to reproduce the other charges of the Standard Model like hypercharge, isospin, ... and all the other internal quantum numbers of elementary particles. This Clifford-algebraic (spinorial) approach to the Standard Model has recently been studied by Budinich [32] to understand how the known internal symmetry groups might derive from the three complex division algebras and to explain the origins of charges and other couplings from a Clifford algebra perspective, not unlike the earlier results obtained by Smith [17] .

After discussing the difference between the Kaluza-Klein compactification and the Clifford approach that avoids the problem of the large value for the electron charge, one can deduce immediately that due to the condition: 


$$
\kappa^{2}-\pi^{2}=\kappa^{2}\left(1-\frac{\pi^{2}}{\kappa^{2}}\right)=m_{P}^{2}\left(1+S^{2}\right)>0
$$

the following ratios are constrained to obey :

$$
0 \leq \frac{\pi^{2}}{\kappa^{2}}=\frac{n^{2} e^{2}}{2} \leq 1 \Rightarrow 0 \leq n^{2} e^{2} \leq 2
$$

which is precisely the same inequality constraint for the values of $n$ found by [13] in their analysis of the Kerr-Newman solutions.

Concluding, for a Planck-mass Clifford polyparticle obeying $\mathcal{M}^{2}=m_{p}^{2}$, the geometric calculus of Clifford algebras in four-dim based on polyvector physical quantities, when one uses the mass-shell condition of the standard relativistic dynamics $p_{\mu} p^{\mu}-m^{2}=0$, and after implementing the spacelike condition on the axialmomentum vector component of the polymomentum $\pi_{\mu} \pi^{\mu}=-\pi^{2}$, leads to the same functional relation among the charge, mass and spin as that based on the Kerr-Newman metric for a rotating-charged black hole, after equating its gravitational horizon radius to the Compton wavelength $\hbar / m_{K N} c$.

This identification simply required to set the extended Planck mass scale $m_{K N}$ to be equal to $\kappa / \sqrt{2}$, where $\kappa$ is the first component of the polymomentum of the polyparticle, and after fixing $\mathcal{M}^{2}=m_{P}^{2}$ in the C-space mass-shell condition $P^{2}=\mathcal{M}^{2}$. After simply rearranging the terms in the latter condition it leads to the sought-after charge-mass-spin relationship derived from eqs- $(24,25,26,27)$.

To sum up, the charge-mass-spin relationship; i.e the relationship between the Clifford-valued quantities of the polymomentum and the Kerr-Newman black hole parameters are:

$$
\begin{gathered}
\kappa^{2}=2 m_{K N}^{2} \cdot \quad-\pi_{\mu} \pi^{\mu}=\pi^{2}=\frac{\kappa^{2} n^{2} e^{2}}{2} . \quad q^{2}=n^{2} e^{2}=n^{2} \alpha . \\
\kappa^{2}-\pi^{2}=\kappa^{2}\left(1-\frac{\pi^{2}}{\kappa^{2}}\right)=m_{P}^{2}\left(1+S^{2}\right)=2 m_{K N}^{2}\left(1-\frac{n^{2} e^{2}}{2}\right) . \\
S^{2}=s^{2}=\frac{1}{2} S^{\mu \nu} S_{\mu \nu} .
\end{gathered}
$$

For finite values of the spin, there is a singularity for the values of $m_{K N}, \kappa, \pi$ in the expression

$$
2 m_{K N}^{2}=\kappa^{2}=\left(m_{P}\right)^{2}\left[\frac{1+S^{2}}{1-\left(n^{2} e^{2} / 2\right)}\right]=\infty .
$$

that occurs when the charge quantization condition saturates its upper bound 


$$
\frac{n^{2} e^{2}}{2}=1 \Rightarrow n^{2} e^{2}=n^{2} \alpha=2 .
$$

Therefore, the charge quantization condition must obey in general the following inequality constraints :

$$
0 \leq \frac{\pi^{2}}{\kappa^{2}}=\frac{n^{2} \alpha}{2} \leq 1
$$

This inequality (38) yields an upper bound in the values of $n=16$ when $\alpha=1 / 128$ and corresponds to the simultaneous singular values of $\pi, \kappa, m_{K N}$.

One could understand the reason why there is an upper bound in eq-(38) for the values of $n=16$ when $\alpha=1 / 137.036<1 / 128$ by noticing that in $D=4$ the degree of the Clifford algebra is $2^{4}=16$, meaning that the number of Clifford-bits is 16 . Hence, the Clifford-polyparticle will carry at most a reasonable value of the charge which is at most 16 times higher than the elecron's charge when the mass parameter $\mathcal{M}$ is equal to the Planck mass $m_{P}$.

To conclude, in essence, the physical meaning of the mass-shell conditon in C-space $P^{2}=\mathcal{M}^{2}$, relating the values of the $\kappa, \pi_{\mu}, S^{\mu \nu}, m, \ldots$ components of the polymomentum is that the true physical mass $\mathcal{M}$ in C-space has a gravitational, electromagnetic, rotational ( spin ), translational ...aspects to it, as one would expect. A different interpretation of the connection among charge, mass and scale-dilations within the context of Scale Relativity has been given by Nottale [22].

\subsection{THE DIRAC ELECTRON AS A KERR-NEWMAN SOLUTION}

This discussion above works well when the masses are of the order of the Planck mass $\mathcal{M}=m_{P}$. However, to describe an ordinary electron requires a different picture because the electron mass is much smaller than the Planck mass. In this case one is going to have in the Kerr-Newman solutions :

$$
m_{K N}^{2}-\frac{q^{2}}{G}-\frac{c^{2} a^{2}}{G^{2}}<0 .
$$

which corresponds to a naked singularity (absence of horizons) as a result that the (repulsive) centrifugal and electric forces strip away the horizon.

These naked singularity Kerr-Newman solutions have been interpreted by Arcos and Pereira as Dirac particles [14] . The Kerr-Newman solution when eq-(39) is obeyed reduces to a naked singularity of circular shape beyond which the metric components fail to be smooth. Hawking-Ellis developed an extended spacetime interpretation by joining one branch of spacetime to another branch via the singular disk, giving rise to a topological nontrivial space. Due to this nontrivial topological structure, the extended Kerr-Newman spacetime admits states with spin 1/2. This extended Kerr-Newman solution was proposed by [14] to be a 
model for the electron-positron systems in which the concepts of mass, charge and spin are all connected with the spacetime geometry. A charge distribution, according to Wheeler, is indistinguishable by an asymptotic observer from a trapped electric flux tube. Mass, therefore, has then a gravitational, rotational and electromagnetic components as argued previously in the definition of the components of the polymomentum of a polyparticle.

Sidharth [21] has given a different interpretation to the the Dirac electron than Arcos and Pereira [14] based also on the Kerr-Newman metric. There are some black hole solutions in string theory which can be interpreted as very massive Planck-mass like elementary particles. This idea goes back to Salam. The importance of the Clifford-polyparticle geomerical interpretation (relationship) among charge, mass and spin is that it is new as far as we know.

\section{FINE STRUCTURE CONSTANT AND CLIFFORD ALGEBRAS}

We have seen how charges can be interpreted as the ratios of the norms of certain components of the polymomentum. In this section we will discuss how the actual electron charge can indeed be written as the ratios of volumes of suitable geometrical spaces obtained from kinematical constraints in momentum spaces associated with two-particle systems [16].

For many years there have been many attempts to find a theoretical basis for the values of the coupling constants of the four forces in Nature and the masses of the fundamental particles without having to invoke the anthropic principle. Most recently Beck [18] has been able to derive the values of all the Standard Model parameters from first principle, including neutrino masses, based on the Kaneko coupled-map lattices in twodimensions (the so-called Chaotic Strings which is the stringy version of the Higgs fields). To our knowledge the first person who presented a formula for the electromagnetic coupling constant was the mathematician Wyler [15]. Smith later on [17], has derived the values of all the couplings and masses of fundamental particles with remarkable precision using hyperdiamond lattices based on discrete Clifford algebras. In particular he reproduced Wyler heuristic formula for the fine structure constant given precisely by the ratios of volumes of geometric spaces:

$$
\alpha=\frac{8 \pi V\left(D_{5}\right)^{1 / 4}}{V\left(S^{4}\right) V\left(\text { Shilov }_{5}\right)}=\frac{9}{8 \pi^{4}}\left(\frac{\pi^{5}}{2^{4} 5 !}\right)^{1 / 4}=\frac{1}{137.03608245}
$$

and which agrees with the observed value of the fine structure constant in five-parts in ten-million.

We have used in (40) the following numerical values for the volumes of the respective spaces: The volume of the 5-dim bounded homogeneous complex domain $D_{5}$ was calculated by Hua to be $\pi^{5} / 2^{4} 5$ ! [20] . The volume of the four-sphere $S_{4}$ is $8 \pi^{2} / 3$ and the volume of the Shilov boundary (of the five-dim complex domain) Shilov 5 whose topology is $R P^{1} \times S^{4}$ is $8 \pi^{3} / 3$. 
The most impending question is what is the connection between all this with Clifford algebras ? Smith [17] already has shown that the hyperdiamond lattices which reproduce the couplings of all fundamental forces and masses are based on discrete Clifford algebras. Gonzalez-Martin has also derived many of the fundamental ratios of particle masses and the Wyler formula for the fine structure constant based on relativistic symmetry groups [ 33 ] and most recently Smilga [16] has derived the Wyler formula based on the Anti de Sitter group $S O(3,2)$ which is the conformal group in 3-dim. The conformal group is just a subgroup of the Clifford group [19] and not surprisingly, the fine structure constant is encoded in the Clifford group via the conformal group $S O(3,2)$ as its subgroup. This is compatible also with Nottale's scale relativity interpretation of the charge-mass relations [22].

Even more, Smilga based his result by noticing that the Inonu-Wigner contraction of a spin $1 / 2$ representation of $S O(3,2)$ yields a translation operator that consists of the usual momentum operator plus the so-called momentum-spin which was comprised of the $\gamma^{\mu}$ Clifford algebra generator ! . This extra contribution to the multi-particle dynamics due to the momentum-spin component $\gamma^{\mu}$ can be described by a perturbation term that precisely has the same structure as the interaction term of Quantum Electrodynamics $e \gamma_{\mu} A^{\mu}$ !. This was the reason why Smilga was able to obtain the value of the fine structure constant based on this extra momentum-spin component. The volume of the parameter space associated with the group $S O(3,2)$ and the multi-particle kinematics delivers a measure for the number of states that can contribute to the interaction term. Hence, the EM coupling constant is given by a geometric probability based on the ratios of volumes of parameter spaces associated with coset spaces ( gauge symmetry groups ). In Smith interpretation [17], the coupling constants are the amplitudes for particles to emit a gauge boson and the masses are the amplitudes to change direction [17] .

Having seen the role of Clifford algebras in Smith derivation [17] of the coupling constants and masses and of the momentum-spin $\gamma^{\mu}$ in Smilga derivation [16] of the fine structure constant, this reinforces our arguments above that charges, like spin, are unified-components of the polymomentum of a polyparticle. Let us probe deeper ino these ideas by looking now at the degree of a Clifford algebra in four spacetime dimensions which has $n=2^{D}=2^{4}$ components or Clifford bits, meaning that the charge ne is at the most 16 times that of the electron The basic inequality of the previous section is saturated when :

$$
n^{2} \alpha=2^{2 D} \alpha=2 \Rightarrow \frac{1}{\alpha}=2^{2 D-1} .
$$

One can interpret eq-(41) as a charge-dimension relationship $f(D)=\frac{1}{\alpha}$ and we can verify from (41) that the value of $D=4$ dimensions is indeed very close indeed to the value $D=4+\epsilon=4.04921$ which is required to yield the numerical value $\frac{1}{\alpha}=137.036$ of the inverse of the fine structure constant. Hence, the 
fractal spacetime dimensionality of $D=4+\epsilon$ seems to be deeply connected to the observed value of the EM coupling constant [29].

\section{C-SPACES AND RENORMALIZATION OF THE ELECTRIC CHARGE}

Using one of the main results of the previous sections is:

$$
\kappa^{2}+\pi_{\mu} \pi^{\mu}=\kappa^{2}-\pi^{2}=m_{P}^{2}\left(1+S^{2}\right)=\text { constant }
$$

which occurs when the values of $S^{2}$ remain constant in the dynamical evolution of the polyparticle, as a function of the C-space proper time $\Sigma$, we can immediately deduce that the values of $\left|\pi_{\mu} \pi^{\mu}\right|$ ( related to the charge ) will increase when the values of $\kappa^{2}$ increase, and vice versa. This is similar to what happens in QED, although the functional relations between charge $e$ and the mass-energy scale $E$ are different. In QED one has for the beta function at one loop, :

$$
\frac{\partial \ln e^{2}}{\partial \ln E}=\frac{e^{2}}{6 \pi^{2}} \Rightarrow e^{2}(E)=\frac{e^{2}\left(E_{0}\right)}{1-\frac{e^{2}\left(E_{0}\right)}{6 \pi^{2}} \ln \left(E / E_{0}\right)} .
$$

which reflects the screening of electric charge with distance as a result of electron-positron pair-creation ( vacuum polarization ) surrounding the bare charge. As the energy increases ( distance decreases) the bare charge increases, and consequently, the fine structure constant increases with energy. There is a Landau charge-singularity $e^{2}=\infty$ when the (finite) energy scale is :

$$
E=E_{0} e^{6 \pi^{2} / e^{2}\left(E_{0}\right)}
$$

whereas previously one has a different situation, the quantity $\left|\pi_{\mu} \pi^{\mu}\right|$ is singular when the energy scale blows up $\kappa=m_{K N} \sqrt{2}=\infty$. However this occurs at a finite saturation point of the quantized electric charge :

$$
2=n^{2} \alpha=n^{2} e^{2}
$$

As we argued before, in four-dimensions, the number of Clifford-bits $n=2^{D}=2^{4}=16$ and the charge is at the most 16 times that of the electron so that $n^{2} / 2=2^{2 D} / 2=2^{8} / 2=2^{7}$, and the last equation establishes a relationship among, dimensions, charge and energy-scale given by :

$$
\frac{1}{2^{7}}=\frac{1}{128}=e^{2}(E)=\frac{e^{2}\left(E_{0}\right)}{1-\frac{e^{2}\left(E_{0}\right)}{6 \pi^{2}} \ln \left(E / E_{0}\right)} .
$$


Such numerical values (47) agrees fully with the Renormalization Group results that fix the $Z$-boson energy scale governing the high-energy radiation in $Z$-boson production/decay and which has been measured to be $1 / 127.934$ and that is very close to $1 / 128[13,17]$.

However, we must exercize caution with the last remarks because the electron mass is much smaller than the Planck mass and this is incompatible with the Planck-mass polyparticle dynamics in C-spaces and the fact that we encounter naked singularities for the Kerr-Newman metric in the electron's case [14]. Because there are no horizons for the electron the above formulae (47) do not longer apply to study the electron. They are valid for a Planck-mass polyparticle whose charge is at most 16 times that of the electron.

In Nottale Scale Relativity, the effective dimensions of fractal-spacetime are resolution-dependent. When the minimal Planck scale resolution is reached the dimensions and energy blow up but the value of the fine structure constant at the Planck scale remains finite and is given by $1 / 4 \pi^{2}[22]$.

Finally, we should mention that if the values of $S^{2}$ were to change in the motion of the polyparticle, as one varies the values of the $\mathrm{C}$-space proper-time parameter $\Sigma$, one will have a different mass-charge evolution behaviour than that given by eq-(42) and the combination $\kappa^{2}-\left|\pi_{\mu} \pi^{\mu}\right|$ is no longer a contant but a function of the C-space proper-time $\Sigma$. It is warranted to explore the physical consequences of this very interesting case when the spin magnitude $S^{2}$ is itself dynamical in the motion of the polyparticle as a function of $\Sigma$, the $\mathrm{C}$-space proper-time parameter, which has the units of a four-dimensional volume and thus is related to scales.

\section{APPENDIX}

The outer and inner horizons radii of the Kerr-Newman metric were used recently by [13] to introduce spin and charge into the definition of a new extended Planck mass scale.

The radii of the inner and outer horizons are given by:

$$
r_{ \pm}=\frac{G}{c^{2}}\left[m_{K N} \pm \sqrt{m_{K N}^{2}-\frac{q^{2}}{G}-\frac{c^{2} a^{2}}{G^{2}}}\right] .
$$

where $q=n e$ is the quantized charge and $a=s \frac{\hbar}{m}$ is the angular momentum per unit mass. The ergosphere is that region where nothing can escape the rotational drag of the rotating black-hole. The horizon is the region where light cannot escape. If one equates the radius $r_{+}$of the outer horizon with the Compton wavelength $\hbar / m_{K N} c$ of the particle of mass $m_{K N}$ one gets :

$$
r_{+}=\frac{\hbar}{m_{K N} c}=\frac{G}{c^{2}}\left[m_{K N}+\sqrt{m_{K N}^{2}-\frac{q^{2}}{G}-\frac{c^{2} a^{2}}{G^{2}}}\right] .
$$


This yields an algebraic relation among mass, charge and spin after solving for $m_{K N}[13]$ :

$$
m_{K N}=\frac{m_{P}}{\sqrt{2}} \sqrt{\frac{2\left(1+s^{2}\right)}{2-\alpha n^{2}}} .
$$

where $m_{P}=\sqrt{\hbar c / G} \sim 2.2 \times 10^{-5}$ grams is the Planck mass and $\alpha=e^{2} / \hbar c$ is the fine structure constant.

Another crucial set of inequalities derived from the choice of the plus sign in front of the square root (A.2) are [13] :

$$
n^{2} e^{2}+s^{2}<1 . \quad n^{2} e^{2}<2 .
$$

Had one chosen the minus sign in front of the square roots in (A.1) one would have arrived at the same mass-spin-charge relation as before ( A.3 ) but the first inequality condition in (A.4) would have been reversed:

$$
n^{2} e^{2}+s^{2}>1 . \quad n^{2} e^{2}<2 .
$$

Notice that the second inequality condition of (A.5) remains the same as before.

In the extreme case scenario, when the $r_{+}=r_{-}$one would have had an strict equality in the first expression of (A.4, A.5) :

$$
n^{2} e^{2}+s^{2}=1 . \quad n^{2} e^{2}<2 .
$$

signaling a balance among the attractive gravitational, and the centrifugal and repulsive electric forces. In this extreme case, the terms inside the square-root of (A.1) are zero and one has that the extended mass scale becomes now the same as the Planck mass:

$$
m_{K N}^{2}=m_{P}^{2} \Rightarrow m_{K N}=m_{P}
$$

\section{ACKNOWLEDGMENTS}

We thank M.Pavsic, T. Smith and J. Mahecha for discussions and to M. Bowers, J. Boedo, T.Smith and D. Banerjee for invaluable assistance in preparing the manuscript.

\section{REFERENCES}


1. D. Hestenes, Spacetime Algebra (Gordon and Breach, New York, 1996). D. Hestenes, G. Sobcyk, Clifford Algebra to Geometric Calculus (D. Reidel Publishing Company, Dordrecht, 1984).

2. I. R. Porteous, Clifford Algebras and the Classical Groups (Cambridge University Press, 1995).

3. W. Baylis, Electrodynamics , A Modern Geometric Approach (Birkhauser, Boston, 1999). G. Trayling and W. Baylis, Journal Phys A 34, 3309 ( 2001 ).

4. B. Jancewicz, Multivectors and Clifford Algebra in Electrodynamics (World Scientific, Singapore 1989).

5. R. Ablamowicz, B. Fauser Clifford Algebras and their applications in Mathematical Physics, Vol 1: Algebras and Physics ( Birkhauser, Boston 2000). J. Ryan, W. Sproessig Clifford Algebras and their applications in Mathematical Physics, Vol 2: Clifford analysis ( Birkhauser, Boston 2000) .

6. P. Lounesto, Clifford Algebras and Spinors (Cambridge University Press 1997).

7-C. Castro, Chaos, Solitons and Fractals 11, 1663 (2000). Foundations of Physics 30, 1301 (2000). Chaos, Solitons and Fractals 11, 1721 (2000). Chaos, Solitons and Fractals 12, 1585 (2001). "The Programs of the Extended Relavity in C-spaces, towards physical foundations of String Theory Advance NATO Workshop on the Nature of Time, Geometry and the Physics of Perception (Kluwer Publishers 2003).

8. W. Pezzaglia, "Physical Applications of a Generalized Geometric Calculus: Papapetrou Equations and Metamorphic curvature [ arXiv : gr-qc/9710027]. "Dimensionally Democratic calculus and Principles of Polydimensional Physics" [ arXiv : gr-qc/9912025] . "Classification of Multivector Theories and Modifications of the Postulates of Physics" [ arXiv : gr-qc/9306006 ].

9. M.Pavsic, The Landscape of Theoretical Physics : A Global View, from Point particles to the Brane world and Beyond, in Search of a Unifying Principle (Kluwer, Dordrecht 2001). Foundations of Physics 31, 1185 (2001). Phys. Let A 242, 187 (1998). Nuovo Cimento A 110, 369 (1997). Foundations of Physics 33, 1277 (2003) .

10. S. Ansoldi, A. Aurilia, C. Castro, E. Spallucci, Phys. Rev. D 64, 026003 (2001) .

11. C. Castro, M. Pavsic, Phys. Lets B 539 , 133 ( 2002).

12. C. Castro, Jour. of Entropy 3, 12 (2001). C. Castro, A. Granik, Foundations of Physics 33 No.3 , 445 (2003).

13. F. I. Cooperstock, V. Faraoini, Mod. Phys. A 18,1037 (2003).

14. H. I. Arcos and J. G. Pereira, "Kerr-Newman solutions as a Dirac particle [ arXiv : hep-th/0210103] .

15. A. Wyler, C. R. Acad. Sc. Paris 269, 743 (1969).

16. W. Smilga, "Higher order terms in the contraction of $S 0(3,2)$ [ arXiv : hep-th/0304137]. 
17. F.D. Smith Jr, Int. J. Theor. Phys 24 , 155 (1985). Int. J. Theor. Phys 25 , 355 ( 1985 ). "From Sets to Quarks [ arXiv : hep-ph/9708379] . also see [ CERN CDS EXT-2003-087 ] .

18. C.Beck, Spatio-Temporal Vacuum fluctuations of Quantized Fields (World Scientific, Singapore 2002 ).

19. C. Castro, M. Pavsic, Int. J. Theor. Phys. 42, 1693 (2003).

20. L.K. Hua, Harmonic Analysis of Functions of Several Complex variables in the Classical Domains ( Birkhauser, Boston, Basel, Berlin 2000 ).

21. B.G. Sidharth, A Chaotic Universe, from the Planck to the Hubble Scale ( Nova Publishers, 2000 ).

22. L. Nottale, Fractal Spacetime and Microphysics, towards the Theory of Scale Relativity (World Scientific, Singapore 1992).

23. P. Ramond, "Exceptional Groups and Physics" [ arXiv : hep-th/0301050].

24. D. Hestenes, R. Ziegler, Acta Applicandae Mathematicae 23, 25 ( 1991 ).

25. C. Castro, Mod. Phys. Lett. A 19 , 19 (2004).

26. M. Defaria-Rosa, E. Recami and W. Rodrigues Phys. Letts B 173, 233 ( 1986 ).

27. H. Pfeiffer, "Higher gauge Theoy and Nonabelian generalization of 2-form Electrodynamics [ arXiv : hep-th/0304074 ]. J. Baez, "Higher Yang-Mills theory" [ arXiv : hep-th/0206130].

28. T. Ivezic, Foundations of Physics Letters 15, 27 ( 2002 ).

29. T.Smith, Private communication.

30. C. Castro, Int. Jour. Mod. Phys. A 18,5445 (2003).

31. C. Castro, M. Pavsic, "The Extended Relativity Theory in Clifford Spaces" submitted to the Int. Jour. Mod. Phys. A (2004).

32. P. Budinich, "Internal Symmetry from Division Algrebras in Pure Spinor Geometry " [ arXiv : hepth/0311045 ] .

G. Gonzalez-Martin, "Physical Geometry " University of Simon Bolivar, publishers, Caracas, June 2000, 265 pages, ISBN : 9800767495. G. Gonzalez-Martin, " The proton/electron Geometric Mass Ratio" [ arXiv : physics/0009052 ] . "The fine structure constant from Relativistic Groups " [ arXiv : physics/0009051 ] 